\title{
ESQUISTOSSOMOSE NA REGIÃO NORTE DO BRASIL
} THE SCHISTOSOMIASIS IN NORTHERN BRAZIL

\author{
Carlos Alberto Rodrigues Junior ${ }^{1,5}$, Fellipe Camargo Ferreira Dias ${ }^{1,5}$, Roniel \\ Thalles Almeida da Silva Rosa ${ }^{1,5}$, Cerize Rodrigues Lima Cardoso ${ }^{2,5}$, Fernanda \\ Paula Fernandes dos Santos Veloso ${ }^{2,5}$, Sandra Maria Botelho Mariano ${ }^{3,5}$, \\ Benta Natânia Silva Figueiredo ${ }^{4,5}$.
}

\section{RESUMO}

A esquistossomose, causada pelo helminto Schistosoma mansoni, é uma doença endêmica no Brasil, apresentando-se como sério problema de saúde pública. Entretanto, apesar da relevância da doença poucos são os estudos sobre a mesma na região Norte do Brasil. Assim, o presente estudo teve por objetivo avaliar as características epidemiológicas da esquistossomose na região entre 2012 e 2015. Foi realizado um estudo transversal das informações coletados no banco de dados oficial do Sistema de Informação de Agravos de Notificação - SINAN, do Ministério da Saúde. Nesse período, foi observada uma tendência no crescimento de casos notificados na região, sendo que $65,09 \%$ foram observados em indivíduos do sexo masculino e $34,91 \%$ do sexo feminino, $71,60 \%$ de raça parda, $17,16 \%$ de raça branca, $71,01 \%$ foram moradores de zona urbana e $26,63 \%$ de zona rural. Além disso, mais da metade da população estudada tinha entre 20 e 59 anos. De acordo com estudos já publicados, há uma tendência de aumento na incidência de infecção humana pelo Schistossoma $s p$. Essa perspectiva de crescimento de casos notificados pode ser observada nos dados encontrados por este estudo, dados relevantes por fornecer informações para construção de políticas de controle e prevenção.

Palavras-chave: Doenças Negligenciadas; Esquistossomose mansônica; Região Norte do Brasil.

Universidade Federal do Tocantins, Tocantins, Brasil; ${ }^{2}$ Mestranda em Ciências da Saúde - Programa de Pós-Graduação em Ciências da Saúde da Universidade Federal do Tocantins, Tocantins, Brasil; ${ }^{3}$ Docente do Curso de Medicina Universidade Federal do Tocantins, Tocantins, Brasil; ${ }^{4}$ Doutoranda em Ciência Animal Tropical - Programa de PósGraduação em Ciência Animal Tropical da Universidade Federal do Tocantins, Tocantins, Brasil; ${ }^{5}$ Laboratório de Experimentação Animal e Biotério Universidade Federal do Tocantins, Tocantins, Brasil.

Autor correspondente: Carlos Alberto Rodrigues Junior; carlos.rouft@gmail.com

Editor: Guedes V. R. Medicina, Universidade Federal do Tocantins, Brasil.

Publicado: 20 de junho de 2017.

Direitos Autorais: (C) 2017 Rodrigues Junior et al. Este é um artigo de acesso aberto que permite o uso, a distribuição e a reprodução sem restrições em qualquer meio, desde que o autor original e a fonte sejam creditados.

Conflito de interesses: os autores declararam que não existem conflitos de interesses.

\section{ABSTRACT}

Schistosomiasis is an endemic disease in Brazil. It is caused by the worm Schistosoma mansoni, presenting itself as a serious public health problem. However, despite the relevance of the disease, there are few studies about the disease in the northern region of Brazil, so the present study aimed to evaluate the epidemiological characteristics of schistosomiasis in the region between 2012 and 2015. A cross-sectional study of the information collected in the bank of the national health information system, of the Ministry of Health. During this period, there was a trend in the growth of reported cases in the region, with $65,09 \%$ observed in males and $34,91 \%$ of the female sex, $71,60 \%$ of the brown race, $17,16 \%$ of the white race, $71,01 \%$ were urban dwellers and $26,63 \%$ were rural. In addition, the majority of the population studied was between 20 and 59 years old. According to previously published studies, there is a trend of increasing incidence of human infection by Schistosoma $s p$. This perspective of growth of reported cases can be observed in the data found by this study, relevant data for providing information for the construction of control and prevention policies.

Keywords: Neglected disease; Esquistossomose mansônica; Northern Brazil. 


\section{INTRODUÇÃO}

A esquistossomose, doença endêmica de origem parasitária desencadeada pelo helminto Schistosoma mansoni, apresenta-se como importante doença no contexto de saúde pública. Estima-se que infecte cerca de 200 milhões de indivíduos e ameace outros 600 milhões que vivem em áreas de risco. Além da ocorrência de formas graves e óbitos, a vasta distribuição geográfica da doença determina a relevância desse problema no contexto brasileiro ${ }^{1}$.

A doença foi descrita pela primeira vez em 1847, porém somente em 1908 foi relatada pela primeira vez no Brasil, no estado da Bahia, apresentando como agente etiológico a espécie $S$. mansoni ${ }^{2,3}$.

Os moluscos do gênero Biomphalaria $s p$. são descritos como hospedeiros intermediários (HI) do parasita, sendo que as espécies $B$. glabrata, $B$. tenagophila e $B$. stramínea são as relatadas como responsáveis por veicular a doença no Brasil ${ }^{4}$. O homem é o hospedeiro definitivo (HD), que ao eliminar os ovos do parasita junto com as fezes, próximo a reservatórios de água doce, contamina o ambiente. Em contato com a água, os ovos eclodem liberando o miracídio, uma larva ciliada responsável por infectar o hospedeiro intermediário. No HI, o miracídio evolui a cercaria, deixando o molusco ativamente após quatro a seis semanas ${ }^{5,6}$.

O homem, ao entrar em contato com águas contendo cercarias, poderá adquirir a parasitose por infecção ativa via pele ou mucosa. No organismo, os parasitas adultos migram e se posicionam no sistema venoso portal onde ocorrerá a reprodução. Os ovos, que geralmente são eliminados nas fezes, poderão gerar reações inflamatórias perivesicais ou peri-intestinais ou embolização em fígado, baço, pulmão e sistema cefalorraquidiano $^{6,7}$.

As manifestações clínicas agudas da doença esquistossomótica são inespecíficas, podendo apresentar mal estar geral, diarreia, fadiga, mialgia, febre e tosse seca, durando de 2 a 10 dias $^{7,8}$. Em casos persistentes e graves, o quadro clínico pode ser composto por perda de peso, dispneia, diarreia, dor abdominal difusa, toxemia, hepatoesplenomegalia e erupções cutâneas ${ }^{7}$.

A região Norte do Brasil é caracterizada como endêmica para doenças parasitárias, principalmente as transmitidas por veiculação hídrica ${ }^{9}$. Diversos autores relatam o avanço da distribuição espacial de moluscos nessa região, aumentando o risco de transmissão de parasitoses, incluindo a esquistossomose ${ }^{10,11}$. Entretanto, são escassos os estudos sobre a doença na região ${ }^{5}$. Nesse sentido, este trabalho teve como objetivo avaliar as características epidemiológicas da esquistossomose na região Norte do Brasil entre 2012 e 2015.

\section{MATERIAL E MÉTODOS}

Foi realizado um estudo transversal com o objetivo de traçar o perfil dos indivíduos acometidos por esquistossomose na região Norte do Brasil no período de 2012 a 2015. Foram coletadas e avaliadas informações presentes no banco de dados oficial do Sistema de Informação de Agravos de Notificação (SINAN), do Ministério da Saúde.

As variáveis analisadas foram idade, sexo, raça e evolução dos casos. As análises exploratórias dos dados foram realizadas a partir da apuração de frequências absolutas e percentuais para as variáveis categóricas.

\section{RESULTADOS}

Analisando-se os dados disponíveis no SINAN entre os anos de 2012 e 2014 foi observada uma tendência no crescimento de casos notificados na região Norte do Brasil, o que não ocorreu no ano de 2015, período com o menor número absoluto de casos $(n=24)$, como pode ser observado na Figura 1. É notório que, dos casos de esquistossomose notificados na região Norte no período estudado, 65,09\% $(n=110)$ correspondia ao sexo masculino e apenas $34,91 \%$ $(n=59)$ ao sexo feminino, no qual a maioria tinha de 20 a 59 anos (68,01\%). Observou-se ainda predominância em indivíduos da raça parda $(71,60 \%)$, seguida da raça branca $(17,16 \%)$. Quanto à zona de residência dos indivíduos afetados, $71,01 \%(n=120)$ relataram serem moradores de zona urbana e $26,63 \%(n=45)$ de zona rural (Tabela 1$)$.

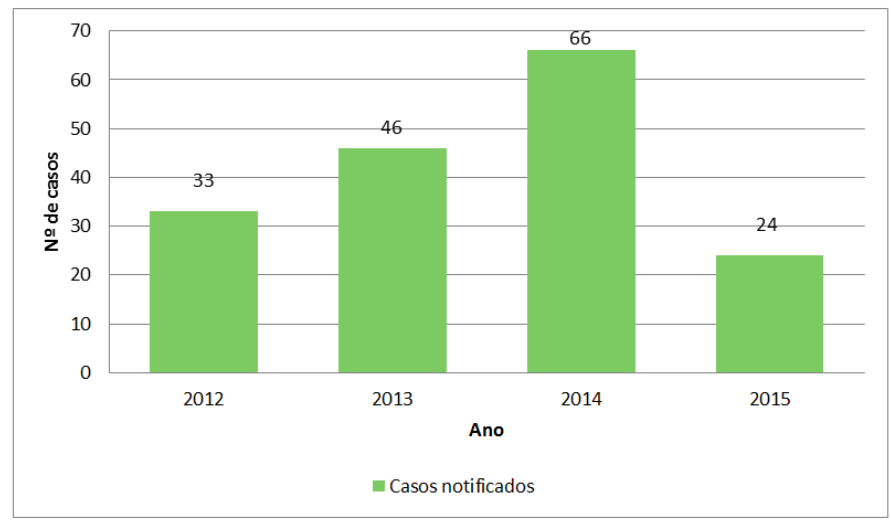

Figura 1: Número de casos notificados de esquistossomose de 2012 a 2015, na região Norte do Brasil.

Tabela 1: Características sociodemográficas dos indivíduos diagnosticados com esquistossomose na região Norte do Brasil, entre 2012 e 2015.

\begin{tabular}{lcc}
\hline Características & $\mathrm{n}$ & $\%$ \\
\hline Sexo & & \\
\hline Masculino & 110 & 65,09 \\
Feminino & 59 & 34,91 \\
\hline Raça & 29 & \\
\hline Branca & 9 & 17,16 \\
Preta & 121 & 5,33 \\
Parda & 1 & 71,60 \\
Amarela & 9 & 0,59 \\
Ignorado/branco & & 5,33 \\
\hline Faixa etária (anos) & 5 & \\
\hline$<1$ & 3 & 2,96 \\
$1-4$ & 4 & 1,78 \\
$5-9$ & 10 & 2,37 \\
$10-14$ & 5 & 5,92 \\
$15-19$ & 55 & 2,96 \\
$20-39$ & 60 & 32,54 \\
$40-59$ & 16 & 35,50 \\
$60-64$ & 4 & 9,47 \\
$65-69$ & 5 & 2,37 \\
$70-79$ & 1 & 2,96 \\
$\geq 80$ & & 0,59 \\
\hline Zona de Residência & 120 & \\
\hline Urbana & 45 & 71,01 \\
Rural & 4 & 26,63 \\
Ignorado/branco & & 2,37 \\
\hline n: número absoluto de casos & & \\
\%: frequência relativa & &
\end{tabular}


Tabela 2: Desfechos dos casos de esquistossomose notificados na região Norte do Brasil entre 2012 e 2015.

\begin{tabular}{|c|c|c|c|c|c|c|c|c|c|c|}
\hline \multirow[t]{2}{*}{ Desfecho dos casos } & \multicolumn{2}{|c|}{2012} & \multicolumn{2}{|c|}{2013} & \multicolumn{2}{|c|}{2014} & \multicolumn{2}{|c|}{2015} & \multicolumn{2}{|c|}{ Total } \\
\hline & $\mathrm{n}$ & $\%$ & $\mathrm{n}$ & $\%$ & $n$ & $\%$ & $\mathrm{n}$ & $\%$ & $\mathrm{n}$ & $\%$ \\
\hline Cura & 18 & 54,55 & 18 & 39,13 & 13 & 19,70 & 5 & 20,83 & 54 & 31,95 \\
\hline Óbito por outra causa & 0 & 0,00 & 0 & 0,00 & 1 & 1,52 & 0 & 0,00 & 1 & 0,59 \\
\hline Ignorado/branco & 15 & 45,45 & 28 & 60,87 & 52 & 78,79 & 19 & 79,17 & 114 & 67,46 \\
\hline
\end{tabular}

Dos indivíduos com diagnóstico de esquistossomose apenas um faleceu no ano de 2014 , entretanto por outras causas. Tiveram a cura registrada $31,95 \%(n=54)$, não havendo descrição do desfecho de $67,46 \%$ dos casos, como demonstrado na Tabela 2.

\section{DISCUSSÃO}

De acordo com estudos já publicados, há uma tendência de aumento na incidência de infecção humana pelo Schistossoma sp., apesar dos esforços da Fundação Nacional de Saúde (FUNASA) para controle desse tipo de doença ${ }^{13}$. Essa perspectiva de crescimento de casos notificados pode ser observada nos dados encontrados por este estudo, tendo em vista que o número de notificações dobrou de 2012 para 2014. Por outro lado, no ano de 2015, apenas 24 casos da doença foram notificados na região Norte, e considerando que não há na literatura relatos de diminuição na incidência ou programa próprio de medidas socioeducativas nessa região, pode-se inferir que há possibilidade de subnotificação nesse ano.

A predominância da esquistossomose na população masculina foi observada neste estudo e corrobora com dados publicados por outros autores ${ }^{13,14,15}$. Embora ambos os sexos estejam submetidos às mesmas condições de saneamento básico, os homens geralmente estão ligados às atividades de pesca e agricultura, em contato direto com água, muitas vezes contaminada, sem nenhum tipo de proteção ${ }^{16}$.

A faixa etária dos indivíduos que procuram serviços de saúde e são diagnosticados com esquistossomose tem relação direta com fases da doença, sendo que em regiões endêmicas, 20 a $40 \%$ dos adultos podem permanecer ativamente infectados ${ }^{6}$.

$\mathrm{Na}$ fase aguda geralmente indivíduos mais novos são diagnosticados, sendo seu principal local de contágio os ambientes de lazer contaminados. Os doentes que possuem uma fase aguda oligossintomática tendem a não buscar atendimento médico e são diagnosticados já na fase crônica devido a algum comprometimento ${ }^{17,18}$. Foi encontrado neste estudo predominância na faixa etária de 40 a 59 anos, corroborando com os achados de Silva et al. ${ }^{13}$ e Jordão et al $^{14}$, em estudos realizados em Pernambuco e Alagoas, respectivamente. Essa maior ocorrência pode ser consequência do contato frequente com água contaminada durante atividades laborais ou de lazer.

A predominância da raça parda nos casos notificados de esquistossomose parece não ter influência direta na fisiopatologia da doença. O fato de a raça ser uma característica autodeclarada, e a parda ser predominante na população brasileira, explica os resultados encontrados neste estudo.

Em relação à zona de residência dos indivíduos parasitados, houve uma predominância de doentes de zona urbana $(71,01 \%)$. Alguns estudos indicam que a maioria da população infectada tem relação direta com a atividade rural, enquanto outros apontam que o ecoturismo tem papel fundamental na história natural da doença e influência na epidemiologia $^{13,19}$. Por apresentar uma predominância em moradores de zona urbana, os dados encontrados sugerem que a população da região Norte infectada com a parasitose teve como momento de contágio situações de lazer e ecoturismo em locais não preparados ou planejados para esse fim.

Além disso, as características climáticas da região e a quantidade de áreas alagadas em toda sua extensão, que favorecem o desenvolvimento do hospedeiro intermediário ${ }^{9}$, associada às precárias condições sanitárias dos municípios da região, possivelmente são fatores determinantes para a presença da parasitose na zona urbana.

A evolução dos doentes com esquistossomose foi favorável nos casos notificados, não sendo relatado nenhum óbito relacionado à doença. A avaliação da taxa de cura ficou comprometida devido à presença de dados incompletos. Em $67,46 \%$ dos casos, esse campo foi ignorado ou deixado em branco, e apenas $31,95 \%$ dos doentes tiveram seu desfecho confirmado como cura no SINAN. Embora seja uma doença negligenciada, a ausência de letalidade nos anos e na região estudada demonstra que mesmo podendo ocorrer formas crônicas e graves, a esquistossomose é passível de tratamento. As políticas públicas de saúde, além de diminuir a morbidade, permitem reduzir a letalidade de doenças infecciosas ${ }^{9}$.

\section{CONCLUSÃO}

Entre os casos notificados de esquistossomose na região Norte do Brasil no período avaliado, predominou indivíduos do sexo masculino, adultos jovens, de raça parda e moradores de zona urbana. Nenhum óbito por esquistossomose foi registrado. Tais dados são relevantes por fornecer informações para construção de políticas de controle e prevenção.

\section{REFERÊNCIAS}

1. Ministério da Saúde (Brasil). Surveillance of Schistosomiasis mansoni in Brazil. Press to Ministry of Health, Diretrizes técnicas. 2014(4): 93p.

2. KATZ, Naftale; ALMEIDA, Karina. Esquistossomose, xistosa, barriga d'água. Cienc. Cult., São Paul, 2003, 55(1): 38-43.

3. Silva P. Contribuição para o estudo da schistosomiase na Bahia. Brasil Médico, 1908, 22:281-283.

4. Fundação Nacional de Saúde. Vigilância e controle de moluscos de importância epidemiológica. In: Secretaria de Vigilância em Saúde. Programa de vigilância e controle da Esquistossomose (PCE): diretrizes técnicas.. 2a ed. Brasília: Ministério da Saúde. No prelo 2007.

5. Pordeus LC. The occurrence of acute and chronic forms of the schistossomiais mansonic in Brazil from 1997 to 
2006: A revision of literature. Epidemiol. Serv. Saúde, Brasília, 2008 17(3): 163-175.

6. COLLEY, D.G.; BUSTINDUY, A.L.; SECOR, W.E.; KING, C.H. 14. Human schistosomiasis. Lancet. Georgia, Estados Unidos. vol. 383, p. 2253-64. 2014.

7. Gryseels B, Polman K, Clerinx J, Kestens L. Human schistosomiasis. Lancet. 2006 Sep 23;368(9541):1106-18.

8. King CH, Dickman K, Tisch DJ. Reassessment of the cost of chronic helmintic infection: a meta-analysis of disabilityrelated outcomes in endemic schistosomiasis. The Lancet. 2005 365(9470): 1561-1569.

9. Marinho JS, de Jesus IM, Asmus CIRF, Lima MO, Oliveira DC. Doenças infecciosas e parasitárias por veiculação hídrica e doenças respiratórias em área industrial, Norte do Brasil. Cad. Saúde Colet., 2016, Rio de Janeiro, 24 (4): 443-451.

10. Valadão $R$, Milward-de-Andrade $R$. Interações de planorbídeos vetores da esquistossomose mansoni e o problema da expansão de endemia na região amazônica. Rev Saúde Públ. 1991 25(5): 353-358.

11. Paraense WL. The Schistosome Vectors in the Americas. Mem Inst Oswaldo Cruz, Rio de Janeiro, 2001, Vol. 96, Suppl.: 7-16.

12. Brasil. SINAN/SVS/MS. Sistema de Informação de Agravos de Notificação - Sinan, Ministério da Saúde, 2017. Disponível em: <http://portalsinan.saude.gov.br/dadosepidemiologicos-sinan>.

13. Silva MBA, Barreto AVMS, Oliveira YV, Bezerra SDC, Bispo BAJ. Perfil epidemiológico de pacientes suspeitos de esquistossomose e patologias associadas em um hospital pernambucano. Rev. Enf. 2015 1(1): 43-46.

Jordão MCC, Macêdo VKB, Lima AF, Junior AFSX. Caracterização do perfil epidemiológico da esquistossomose no estado de alagoas. Ciências Biológicas e da Saúde 2014 2(2): 175-188.

15. SIQUEIRA LMV. Evaluation of parasitological and molecular techniques for the diagnosis and assessment of cure of schistosomiasis mansoni in a low transmission area. Memórias do Instituto Oswaldo Cruz 2015 110(2): 209-214.

16. Araújo KCGM, Resendes APC, Souza-Santos R, Silveira Júnior JC, Barbosa CS. Análise Espacial do focos de Biomphalariaglabrata e de casos humanos de esquistossomose mansônica em Porto de Galinhas, Pernambuco, Brasil, no ano 2000. Cad. Saúde Pública, 2001 23(2):409-417.

17. Santos AM, Melo ACFL. Prevalência da esquistossomose num povoado do Município de Tutóia, Estado do Maranhão. Rev. Soc. Bras. Med. Trop. [online]. 2011, vol.44, n.1, pp. 97-99. ISSN 0037-8682.

18. Resendes APC, Souza-Santos R, Barbosa CS. Internação hospitalar e mortalidade por esquistossomose mansônica no Estado de Pernambuco, Brasil, 1992/2000. Cad. Saúde Pública. Rio de Janeiro, 2005, p. 1392-1401.

19. Massara CL, Amaral GL, Caldeira RL, Drummond SC, Enk MJ, Carvalho OS. Esquistossomose em área de ecoturismo do Estado de Minas Gerais, Brasil. Cad. Saúde Pública, Rio de Janeiro, 2008 24(7):1709-1712. 